\title{
Functional Expression and Characterization of Acetyl Xylan Esterases CE Family 7 from Lactobacillus antri and Bacillus halodurans
}

\author{
Min-Jeong Kim, Myoung-Uoon Jang, Gyeong-Hwa Nam, Heeji Shin, Jeong-Rok Song, and Tae-Jip Kim* \\ Division of Animal, Horticultural and Food Sciences, Graduate School of Chungbuk National University, Cheongju 28644, Republic of Korea
}

\author{
Received: January 3, 2020 \\ Revised: January 14, 2020 \\ Accepted: January 15, 2020 \\ First published online: \\ January 17, 2020 \\ *Corresponding author \\ Phone: +82-43-261-3354 \\ Fax: +82-43-271-4412 \\ E-mail: tjkim@cbnu.ac.kr \\ pISSN 1017-7825, eISSN 1738-8872 \\ Copyright(C) 2020 by \\ The Korean Society for Microbiology \\ and Biotechnology
}

Acetyl xylan esterase (AXE; E.C. 3.1.1.72) is one of the accessory enzymes for xylan degradation, which can remove the terminal acetate residues from xylan polymers. In this study, two genes encoding putative AXEs (LaAXE and BhAXE) were cloned from Lactobacillus antri DSM 16041 and Bacillus halodurans C-125, and constitutively expressed in Escherichia coli. They possess considerable activities towards various substrates such as $p$-nitrophenyl acetate, 4-methylumbelliferyl acetate, glucose pentaacetate, and 7-amino cephalosporanic acid. LaAXE and BhAXE showed the highest activities at $\mathrm{pH} 7.0$ and 8.0 at $50^{\circ} \mathrm{C}$, respectively. These enzymes are AXE members of carbohydrate esterase (CE) family 7 with the cephalosporine-C deacetylase activity for the production of antibiotics precursors. The simultaneous treatment of LaAXE with Thermotoga neapolitana $\beta$-xylanase showed 1.44-fold higher synergistic degradation of beechwood xylan than the single treatment of xylanase, whereas BhAXE showed no significant synergism. It was suggested that LaAXE can deacetylate beechwood xylan and enhance the successive accessibility of xylanase towards the resulting substrates. The novel LaAXE originated from a lactic acid bacterium will be utilized for the enzymatic production of D-xylose and xylooligosaccharides.

Keywords: Acetyl xylan esterases, carbohydrate esterase (CE) family 7, synergistic xylan degradation, Lactobacillus antri, Bacillus halodurans

\section{Introduction}

Xylans are one of the most abundant hemicellulosic bioresources and are widely distributed in plants. These $\beta$ $(1,4)$-linked D-xylanopyranosyl polymers possess various side chains being substituted with L-arabinose, $\alpha$-glucuronic acid, acetate, and ferulic acid residues. Therefore, the complete xylan degradation requires the cooperative actions of several accessory enzymes with the major xylan hydrolases such as endo- $\beta$-xylanases [1-4].

Acetyl xylan esterase (AXE, E.C. 3.1.1.72) is the xylanolytic accessory enzyme hydrolyzing the ester linkages of xylans and/or acetylated sugars to remove the acetate residues. The deacetylating activity of AXE can facilitate the hydrolytic actions of $\beta$-xylanase to produce higher amounts of D-xylose and xylooligosaccharides (XOSs) from agricultural wastes [4, 5]. The resulting products are utilized as functional sugar substitutes for diabetics and as fermentable sugars for bioethanol production [4, 6].

In the carbohydrate active enzyme database (CAZy; http://www.cazy.org), AXEs are categorized to the carbohydrate esterase (CE) families $1 \sim 7,12$, and 16, on the basis of their amino acid sequence similarity, substrate specificity, and protein structure [5]. A number of AXEs have been studied from various bacteria including Bacillus, Thermoanaerobacterium, Streptomyces, Aspergillus, Trichoderma, and Thermotoga species [7, 8]. The majority of AXEs are the serine-type esterases with activity on small substrates such as 4-MUAc and $p$-NPAc. Among them, some microbial AXEs with cephalosporin-C deacetylase (CCD; E.C. 3.1.1.41) activity have been categorized to CE family 7 [5]. The microbial AXEs CE7 have been reported from B. subtilis SHS0133 [9], B. pumilus PS213 [10], Thermotoga maritima [11], B. subtilis CICC20034 [12], and so on. These enzymes could produce $\beta$-lactam antibiotics by using the deacetylated cephalosporin or 7-aminocephalosporanic acid (7-ACA) as 
the intermediates $[13,14]$.

Similar to the approved prebiotics of fructooligosaccharides (FOSs) and galactooligosaccharides (GOSs), XOSs with a degree of polymerization of $2 \sim 10$ are considered as prebiotic candidates promoting the growth of probiotics, Bifidobacterium [15] and Lactobacillus species [16]. The health-beneficial effects of XOSs are a wide range of biological functions including antioxidant, antimicrobial, antiallergy, antiinfection, anti-inflammatory, and immuno-modulatory activities [6]. Nevertheless, only a few studies about the synergistic effects of AXEs and $\beta$-xylanases have been done to date.

In the present study, a putative gene encoding AXE CE7 has been firstly expressed and characterized from Lactobacillus antri, a lactic acid bacterium. As a reference model enzyme, an AXE CE7 from Bacillus halodurans was also cloned and functionally expressed in E. coli. Especially, their CCD activities and synergistic effects on beechwood xylan degradation with $\beta$-xylanase were comparatively characterized with the other known microbial AXEs.

\section{Materials and Methods}

\section{Enzymes and Reagents}

A DNeasy Blood/Tissue Kit (Qiagen, Germany) was used for the preparation of bacterial genomic DNA. Oligonucleotide primers, an Accuprep Plasmid Extraction Kit and a PCR Purification Kit were supplied by Bioneer (Korea). The restriction endonucleases and Pyrobest DNA polymerase were purchased from Takara Biomedical (Japan). Various substrates of $p$ nitrophenyl acetate ( $p$-NPAc), 4-methylumbelliferyl acetate (4MUAc), $\beta$-D-glucose pentaacetate (GPAc), 7-aminocephalosporanic acid (7-ACA), and beechwood xylan (BEX) were obtained from Sigma-Aldrich (USA).

\section{Gene Cloning of AXEs and XN}

The genomic DNA templates were prepared from the cultures of Lactobacillus antri DSM 16041 (=KCTC 5855) and Bacillus halodurans C-125 grown in nutrient broth $(0.5 \%$ peptone, $0.3 \%$ meat extract, and $1.0 \% \mathrm{MnSO}_{4}$ ) at $30^{\circ} \mathrm{C}$ and in Luria-Bertani (LB) broth $(1.0 \%$ tryptone, $1.0 \%$ sodium chloride, and $0.5 \%$ yeast extract) at $37^{\circ} \mathrm{C}$, respectively. The sets of PCR primers, LaAXE-N (5'-TTTTCATATGCAAGATATTCAGCAAT- $\left.3^{\prime}\right)$, LaAXE-C (5'TTTTCTCGAGGTCTTTGCCAATTTGAAT-3'), BhAXE-N (5'TTTTCATATGCCACTAATAGACATG-3'), and BhAXE-C (5'TTTTCTCGAGGAGATCAGATAAAAATTGAA-3') were used for the gene amplification of LaAXE (GenBank ID: EEW54661.1) and BhAXE (BAB07045.1), respectively. PCR was carried out using a C-1000 thermal cycler (Bio-Rad, Hercules, USA) for 30 cycles at $98^{\circ} \mathrm{C}$ for $30 \mathrm{sec}, 50 \sim 52^{\circ} \mathrm{C}$ for $30 \mathrm{sec}$, and $72^{\circ} \mathrm{C}$ for $60 \mathrm{sec}$. The PCRamplified fragment was digested with NdeI and Xhol, and cloned into an expression vector, $\mathrm{pHCXHD}$ [17]. The resulting recombinant plasmids were designated as pHCLaAXE and pHCBhAXE, respectively. For the gene cloning of $\beta$-xylanase (TnXNB; AAN16480.1) from Thermotoga neapolitana DSM 4359, the corresponding gene was amplified by using a pair of PCR primers, TnXNB-N (5'TTTTGAATTCATGAAAGGGTTGCCTGC- $\left.3^{\prime}\right)$ and TnXNB-C (5'TTTTCTCGAGTCTTTCCTTCAGTTTTTCCT- ${ }^{\prime}$ ). The amplified fragment digested with EcoRI and XhoI was cloned into PHCXHD, which was designated as $\mathrm{pHCTnXNB}$.

\section{Gene Expression and Enzyme Purification}

Each recombinant E. coli MC1061 harboring pHCLaAXE, pHCBhAXE, or pHCTnXNB was grown in LB broth containing $100 \mu \mathrm{g} / \mathrm{ml}$ of ampicillin at $37^{\circ} \mathrm{C}$ for $12 \mathrm{~h}$. E. coli cells were disrupted by ultrasonicator VCX750 (Sonics \& Materials, USA). The recombinant enzyme with C-terminal six-histidines was purified by using Ni-NTA affinity chromatography (Qiagen). The purity and the molecular mass of each enzyme were determined by using $12 \%$ SDS-PAGE analyses. The protein concentration was measured using the BCA Protein Assay Kit (Pierce Biotechnology, USA) with bovine serum albumin as a standard.

\section{AXE Activity Assays}

For the activity assay on $p$-NPAc, $1 \mathrm{mM}$ of $p$-NPAc was reacted with AXE and the absorbance of $p$-nitrophenol released was measured at $405 \mathrm{~nm}$. For the activity assay on 4-MUAc, the resulting absorbance was measured at $354 \mathrm{~nm}$ from $0.4 \mathrm{mM}$ of substrate. One unit of AXE activity on $p$-NPAc or 4-MUAc was defined as the enzyme amount releasing $1 \mu \mathrm{mol}$ of each hydrolyzed product per min. In order to determine the AXE activity towards GPAc or 7-ACA, each AXE was reacted with $20 \mathrm{mM}$ of each substrate. The resulting acetate concentration was measured by high-performance liquid chromatography (HPLC) Acme 9000 (Young-Lin, Korea) with UV detector at $210 \mathrm{~nm}$, Aminex HPX-87H column (300 mm x $7.8 \mathrm{~mm}$, Bio-Rad), and $0.6 \mathrm{ml} / \mathrm{min}$ of $0.008 \mathrm{~N}$ sulfuric acid as a mobile phase. One unit of AXE activity on each substrate was defined as the enzyme amount for liberating $1 \mu \mathrm{mol}$ of acetate equivalent per min.

\section{Xylanase Activity Assay and Synergistic Effect Analysis}

The 3,5-dinitrosalicylic acid (DNS) reducing sugar assay was used to determine the TnXNB activity on $2.5 \%$ of BEX, and the absorbance of reducing sugar was measured at $550 \mathrm{~nm}$. One unit of enzyme activity was defined as the TnXNB amount for producing $1 \mu \mathrm{mol}$ of reducing sugar as D-xylose equivalent per $\mathrm{min}$. In order to examine the synergistic effects of the simultaneous treatment with TnXNB and AXE, $2.5 \%$ of BEX was reacted with TnXNB ( $8 \mathrm{mU} / \mathrm{ml}$ on BEX) and each AXE (2 U/ml on 4-MUAc) under the controlled reaction condition. For the stepwise enzyme treatment, each AXE was reacted with $2.5 \%$ of BEX for $4 \mathrm{~h}$ and then inactivated by boiling for $5 \mathrm{~min}$. The resulting reaction mixture was further treated with TnXNB for an additional $4 \mathrm{~h}$. The amount of reducing sugar liberated was determined by DNS assay. LaAXE and BhAXE were reacted with BEX in $50 \mathrm{mM}$ 
sodium acetate $\left(\mathrm{pH} \mathrm{7.0)}\right.$ at $60^{\circ} \mathrm{C}$ and in $50 \mathrm{mM}$ Tris- $\mathrm{HCl}(\mathrm{pH} 7.0)$ at $50^{\circ} \mathrm{C}$, respectively.

\section{Thin-Layer Chromatography Analysis}

Thin-layer chromatography (TLC) was employed for the timecourse analysis of GPAc hydrolysis. $0.1 \mathrm{U} / \mathrm{ml}$ of each AXE was reacted with $50 \mathrm{mM}$ of GPAc under its optimal conditions for each time period. The resulting hydrolysis products were separated on a $60 \mathrm{~F}_{254}$ silica gel plate (Merck) using the solvent mixture of ethylacetate:benzene:isopropanol (2.0:1.0:0.1). The spots were detected by dipping the plate in the developing solution $(0.3 \% \mathrm{~N}$ 1-naphthyl-ethylenediamine and $5 \% \mathrm{H}_{2} \mathrm{SO}_{4}$ in methanol), and then heating it at $110^{\circ} \mathrm{C}$ for $10 \mathrm{~min}$.

\section{Results and Discussion}

\section{Gene Cloning and Expression of AXEs}

It was reported that a marine isolate, Lactobacillus sp., showed xylanolytic enzyme activities such as $\alpha$-Larabinofuranosidase and AXE [18]. However, the related genes and enzymes have not been characterized from lactic acid bacteria yet. In the present study, the gene encoding a putative AXE (LaAXE) has been found for the first time from the genome of Lactobacillus antri DSM 16041 [19]. The structural gene of LaAXE (GenBank ID: EEW54661.1) consisting of $972 \mathrm{bp}$ encoding 323 amino acids $(37 \mathrm{kDa})$ was PCR-amplified and cloned into an expression vector of pHCXHD, which was designated as pHCLaAXE (4,695 bp). LaAXE showed below $38 \%$ of amino acid sequence identities with the other known bacterial AXEs [7, 8]. As a reference AXE enzyme, the other putative gene encoding BhAXE (BAB07045.1; 319 amino acids and $36 \mathrm{kDa}$ ) was also cloned from an alkaliphile, B. halodurans C-125 [20]. BhAXE shares the highest amino acid sequence identity (37.8\%) with LaAXE. Most of the AXEs from Bacillus spp. were known to possess not only AXE activity, but also CCD activity. On the contrary, LaAXE showed much lower sequence identities (below 15\%) to the AXEs from Trichoderma reesi [21] and Aspergillus oryzae [22]. These fungal AXEs were reported to possess only AXE activity without CCD activity.

The recombinant LaAXE or BhAXE with C-terminal sixhistidines was constitutively expressed and purified from E. coli harboring $\mathrm{pHCLaAXE}$ or $\mathrm{pHCBhAXE}$ via Ni-NTA affinity chromatography (Fig. 1). The gel permeation chromatography analyses showed that the native LaAXE and BhAXE are likely to exist as the homo-trimeric or tetrameric forms (apparent molecular mass of 127 and $105 \mathrm{kDa}$, respectively) in solution (Fig. 2). It was revealed
A
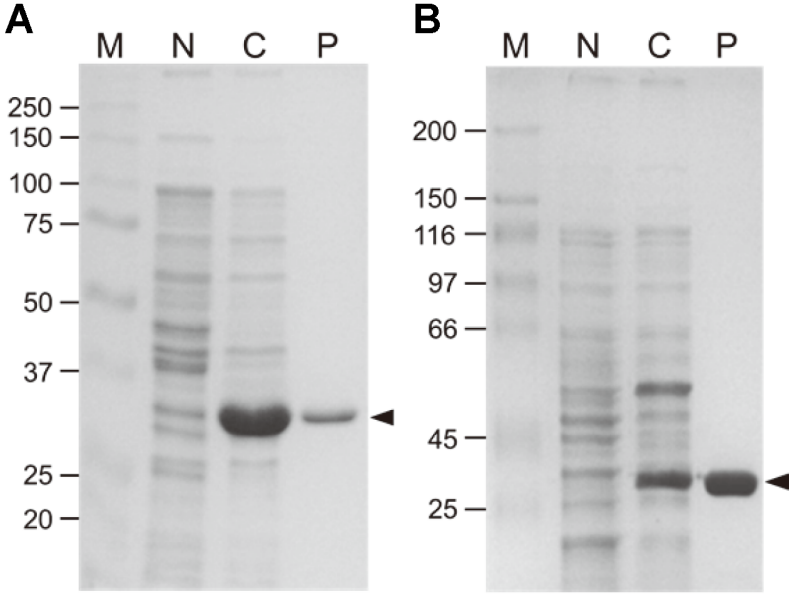

Fig. 1. SDS-PAGE analysis for gene expression and enzyme purification of LaAXE (A) and BhAXE (B) from recombinant E. coli.

Lanes M, protein size marker; $\mathrm{N}$, cell extract from E. coli harboring an empty vector ( $\mathrm{pHCXHD)}$ as a negative control; $\mathrm{C}$, cell extract from $E$. coli containing AXE gene; $\mathrm{P}$, recombinant AXE purified by Ni-NTA chromatography. The AXE bands are indicated by arrowheads.

that the representative AXE CE family 7 from B. pumilus has the doughnut-like hexameric conformation [23]. Another highly thermostable AXE CE7 from T. maritima was a homo-hexamer in solution, but a dimeric form was also observed [24].

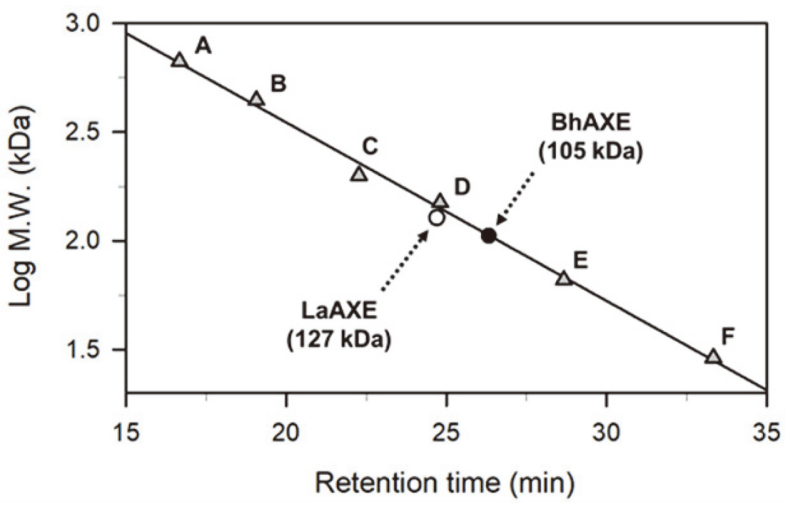

Fig. 2. Quaternary structure estimation of AXEs by gel permeation chromatography with Superdex 200 column.

The molecular weights of LaAXE and BhAXE were indicated by open and closed circles, respectively. Protein molecular weight standards (triangles) consist of thyroglobulin (A, $669 \mathrm{kDa})$, Apoferritin (B, $443 \mathrm{kDa}), \beta$-amylase $(\mathbf{C}, 200 \mathrm{kDa})$, alcohol dehydrogenase $(\mathbf{D}, 150 \mathrm{kDa})$, bovine serum albumin $(\mathbf{E}, 66 \mathrm{kDa})$, and carbonic anhydrase (F, $29 \mathrm{kDa})$. 

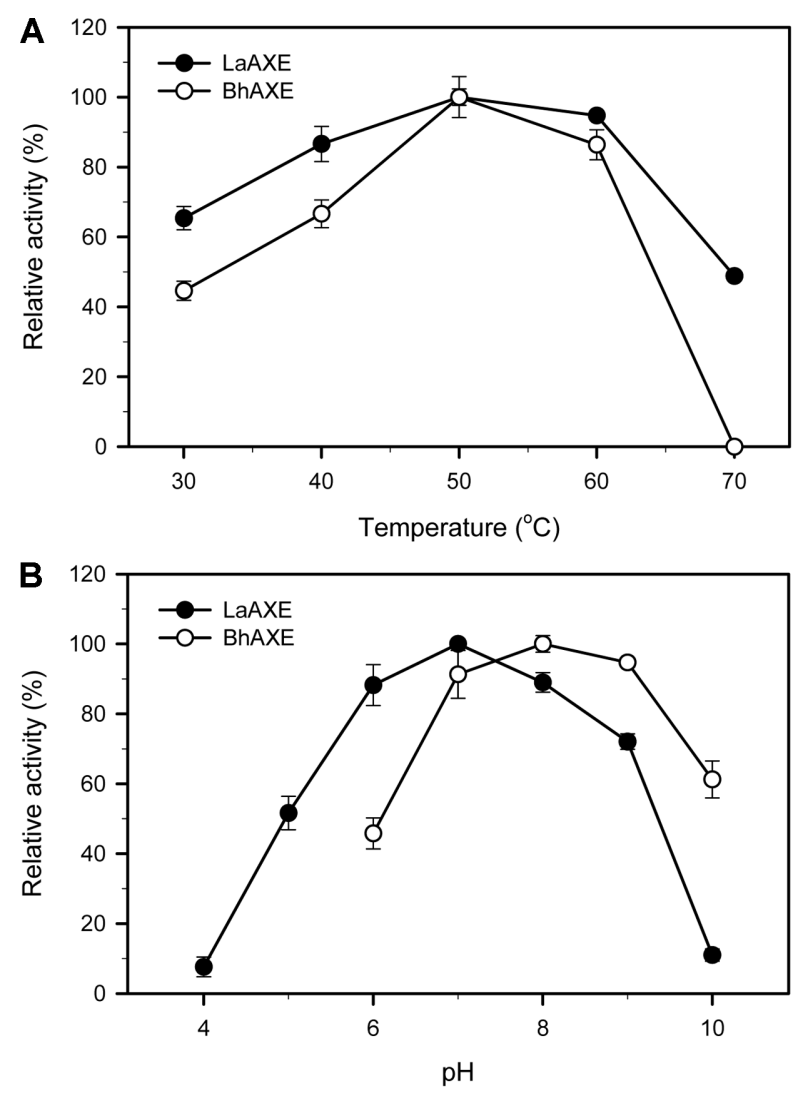

Fig. 3. Effects of temperature (A) and $\mathrm{pH}(\mathbf{B})$ on the activities of LaAXE and BhAXE.

Relative activities of AXEs on glucose pentaacetate (GPAc) were determined at different temperature and $\mathrm{pH}$ conditions.

\section{Enzymatic Characterization of AXEs}

Due to the low stability of 4-MUAc at the alkaline $\mathrm{pH}$ above 9.0, the optimal reaction conditions of LaAXE and BhAXE were determined by using HPLC analysis with GPAc as a substrate. Both AXEs showed the highest activities at $50^{\circ} \mathrm{C}$ in $50 \mathrm{mM}$ sodium phosphate buffer at $\mathrm{pH}$ 7.0 (Fig. 3A). At the high temperature of $70^{\circ} \mathrm{C}$, the activity of LaAXE decreased to $48.8 \%$, whereas BhAXE lost almost all its activity. LaAXE and BhAXE showed the highest activities in $50 \mathrm{mM}$ sodium phosphate buffer ( $\mathrm{pH}$ 7.0) and Tris- $\mathrm{HCl}$ buffer ( $\mathrm{pH}$ 8.0), respectively (Fig. 3B). While LaAXE was stable at the broad $\mathrm{pH}$ ranges between 6.0 and 11.0 , only less than $30 \%$ of its activity remained at the $\mathrm{pH}$ ranges below 5.0 or above 12.0. BhAXE showed high stability at the $\mathrm{pH}$ ranges between 7.0 and 10.0 (data not shown). The common microbial AXEs possess their optimal reaction conditions at the $\mathrm{pH}$ ranges of $6.0 \sim 8.0$ and at temperatures from 30 to $70^{\circ} \mathrm{C}[7,8]$. The highly thermostable AXEs were reported from the thermophiles such as $T$. maritima [24], Thermoanaerobacterium sp. [25], and Thermobifida fusca [26]. The fungal AXEs from Aspergillus oryzae [22] and Trichoderma reesei [21] are the mesophilic enzymes that are highly active at $45 \sim 65^{\circ} \mathrm{C}$, whereas a cold-active AXE was also reported from Paenibacillus sp. [27].

\section{Substrate Specificities of AXEs}

In order to determine the substrate specificity of AXEs, the enzyme reactions were conducted at its optimal reaction conditions with various substrates including GPAc, 4-MUAc, $p$-NPAc, and 7-ACA. As shown in Table 1, LaAXE showed the highest specific activity of $499.5 \mathrm{U} / \mathrm{mg}$ on GPAc. Among the synthetic substrates, $p$-NPAc is the much better substrate for LaAXE than 4-MUAc. Expectedly, it was found that LaAXE possesses the detectable deacetylating activity on 7-ACA (20.7 U/mg), even though this activity is only $4.1 \%$ of that on GPAc. Meanwhile, BhAXE showed the highest specific activity of $501.0 \mathrm{U} / \mathrm{mg}$ on GPAc. Especially, it has 8.0 times higher activity on 7ACA (165.0 U/mg) than LaAXE, which corresponds to $32.9 \%$ of the activity towards GPAc.

In general, the hemicellulose-deacetylating AXEs are members of CE families 1 7 [8]. The substrate preferences of AXEs are highly dependent on the microbial origins. For example, the AXE from Bacillus subtilis CICC20034 showed the highest activity $(2,949.0 \mathrm{U} / \mathrm{mg})$ on $p$-NPAC, whereas its relative activities on GPAc and 4-MUAc are 42.2 and 36.8\% of that on $p$-NPAc, respectively [12]. On the other hand, GPAc is the most preferred substrate for the AXE from $B$. pumilus PS213 [10].

As LaAXE and BhAXE showed the considerable deacetylating activities on 7-ACA, these enzymes could be categorized into the AXEs CE family 7. The cephalosporin$\mathrm{C}$ deacetylases (CCDs) are the carbohydrate esterases which hydrolyze the ester linkages of the acetyl groups of 7-ACA as well as the acetylated xylans [5, 12]. BhAXE showed the specific activity of $165.0 \mathrm{U} / \mathrm{mg}$ on 7 -ACA,

Table 1. Specific activities of AXEs on various acetylated substrates.

\begin{tabular}{lcc}
\hline \multirow{2}{*}{ Substrates $^{\mathrm{a}}$} & \multicolumn{2}{c}{ Specific activity $(\mathrm{U} / \mathrm{mg})$} \\
\cline { 2 - 3 } & LaAXE & BhAXE \\
\hline GPAc & $499.5 \pm 6.3(100.0 \%)$ & $501.0 \pm 20.0(100.0 \%)$ \\
4-MUAc & $48.7 \pm 0.7(9.7 \%)$ & $293.0 \pm 0.0(58.5 \%)$ \\
- -NPAc & $202.9 \pm 6.6(40.6 \%)$ & $134.6 \pm 2.4(26.9 \%)$ \\
7-ACA & $20.7 \pm 0.6(4.1 \%)$ & $165.0 \pm 0.7(32.9 \%)$ \\
\hline
\end{tabular}

${ }^{\mathrm{a} G P A c}, \beta$-D-glucose pentaacetate; 4-MUAc, 4-methyl umbelliferyl acetate; $p$ NPAc, $p$-nitrophenyl acetate; 7-ACA, 7-aminocephalosporanic acid 


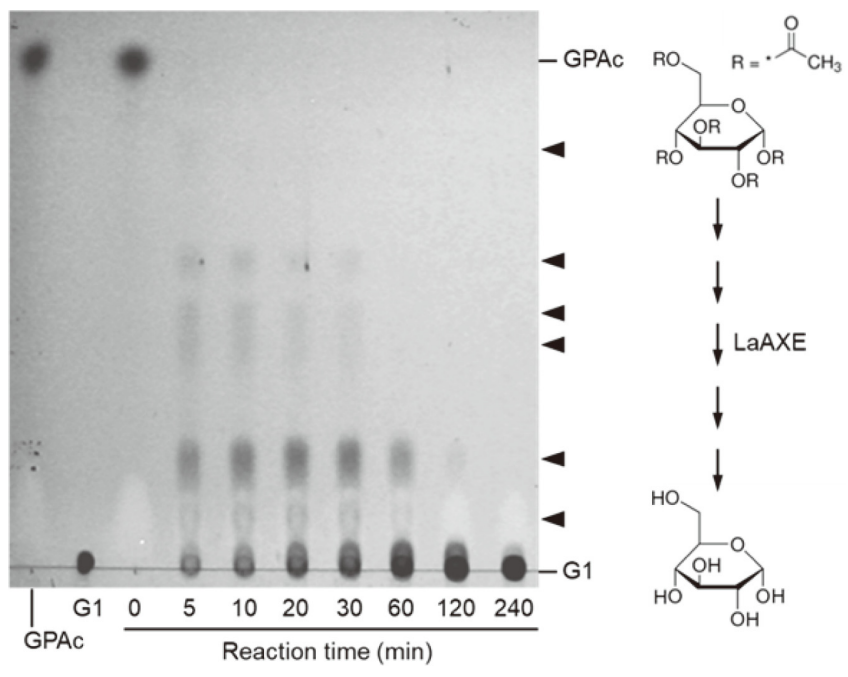

Fig. 4. Time-course TLC analysis of glucose pentaacetate (GPAc) deacetylation catalyzed by LaAXE.

$0.1 \mathrm{U} / \mathrm{ml}$ of enzyme was reacted with $50 \mathrm{mM}$ GPAc in $50 \mathrm{mM}$ sodium phosphate buffer $(\mathrm{pH} 7.0)$ at $50^{\circ} \mathrm{C}$. The putative deacetylated intermediates from GPAc to glucose (G1) are indicated by the arrowheads.

which is similar with that of CCD from Bacillus pumilus PS213 [10].

GPAc contains a total of five acetate groups linked to C$1, \mathrm{C}-2, \mathrm{C}-3, \mathrm{C}-4$, and C-6 of glucose. The time-course study was performed to investigate the deacetylation patterns of GPAc by AXEs. When LaAXE was reacted with GPAc, a series of deacetylated intermediates were detected by TLC analysis (Fig. 4). BhAXE showed almost identical deacetylation patterns of GPAc to LaAXE. Although the exact chemical structures of each intermediate compound were not identified, LaAXE and BhAXE are likely to catalyze the stepwise deacetylation of GPAc to produce only glucose as an end-product. Their detailed positional specificity AXE should be further investigated.

\section{Synergistic Effect on Xylan Degradation}

AXE can remove the acetate groups from the heteroxylans, which facilitates the successive actions of main xylanhydrolases such as $\beta$-xylanase and $\beta$-xylosidase [4]. The synergistic effects of AXEs on the xylan degradation were investigated with $\beta$-xylanase. $\beta$-Xylanase from Thermotoga neapolitana DSM 4359 (TnXNB, GenBank ID: AAN16480.1) is one of the typical endo-hydrolases, which efficiently hydrolyzes beechwood xylan (BEX) to produce xylobiose and xylotriose as the major products [28]. The simultaneous treatment of LaAXE and TnXNB showed the maximal
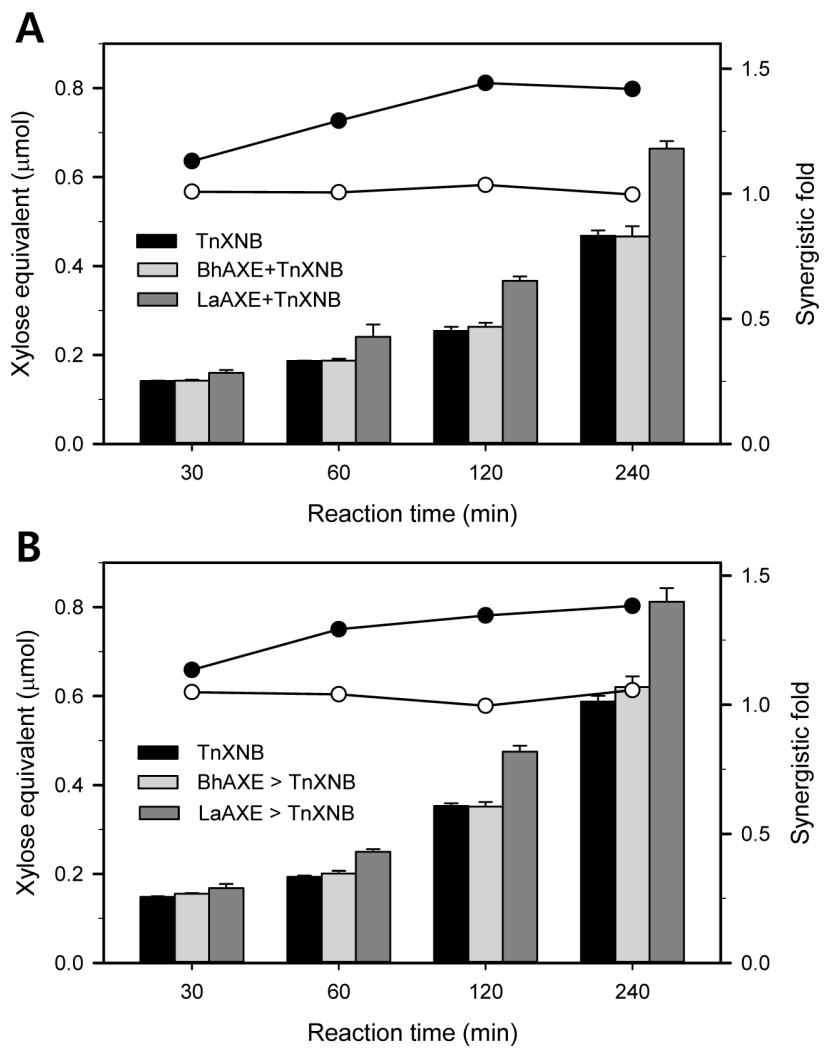

Fig. 5. Synergistic degradation of beechwood xylan (BEX) via the simultaneous (A) and the stepwise (B) treatments of AXE and TnXNB.

$\operatorname{TnXNB}(8 \mathrm{mU} / \mathrm{ml})$ and each AXE $(2.0 \mathrm{U} / \mathrm{ml})$ were reacted on $2.5 \%$ of $\mathrm{BEX}$ in $50 \mathrm{mM}$ sodium acetate $(\mathrm{pH} 5.5)$ at $60^{\circ} \mathrm{C}$ with LaAXE and Tris$\mathrm{HCl}(\mathrm{pH} 7.0)$ at $50^{\circ} \mathrm{C}$ with BhAXE for $4 \mathrm{~h}$, respectively. The xylose equivalent was measured by DNS reducing sugar assay. The synergistic folds were shown as the solid lines with the symbols of closed (LaAXE) and open (BhAXE) circles, respectively.

synergistic fold of 1.44 for $2 \mathrm{~h}$ (Fig. 5A). The stepwise treatment of LaAXE to TnXNB also showed the highest synergistic fold of 1.38 for $4 \mathrm{~h}$ (Fig. 5B). Although the simultaneous treatment showed slightly higher synergistic fold than the stepwise treatment, the difference between them seemed negligible. These results implied that LaAXE could attack both oligomeric and polymeric BEX to generate the deacetylated substrates. TnXNB could easily hydrolyze the resulting deacetylated substrates to shorter products. In case of Volvariella volvacea, the stepwise treatment of AXE to $\mathrm{XN}$ showed the higher synergistic fold of 1.4 than the simultaneous treatment of 1.1-fold [2]. The simultaneous treatments of AXE and XN from Streptomyces sp. and Thermobifida fusca on oat-spelt xylan showed the 
highest synergistic folds of 1.3 [1]. The AXE from Ochrovirga pacifica showed 1.4-fold of synergism on BEX with a commercial xylanase [3].

Unexpectedly, the maximal synergistic folds of BhAXE and TnXNB were 1.03 and 1.06 by the simultaneous and the stepwise treatments, respectively (Fig. 5). Even though the reasons for these differences between them have not been unveiled yet, the modes of action of each AXE were obviously distinguished from the other. The reasons why BhAXE cannot make significant synergistic effects on BEX degradation should be further investigated with the different types of xylans and $\beta$-xylanases. These considerable synergistic actions of LaAXE can be utilized for the efficient degradation of xylan polymers to produce the functional carbohydrates, D-xylose and/or XOSs.

\section{Enzyme Classification of AXEs}

Various types of semi-synthetic $\beta$-lactam antibiotics, such as cefoxitin and cefuroxime, require the deacetylation of cephalosporin-C or 7-ACA as the precursor compounds [13]. To date, the AXE CE7 from B. subtilis CICC 20034 with the highest deacetylating activity on 7-ACA (888 U/mg) was developed for the large-scale enzymatic production of the deacetylated 7-ACA [12]. The improved activity and specificity of AXE CE7 or CCD should be needed for the efficient industrial production of deacetyl cephalosporin-C and 7 -ACA $[12,14]$.

The genes encoding AXEs and the CCDs may be divergently evolved from a common ancestor belonging to the CE family 7. The phylogenetic analyses revealed that both LaAXE and BhAXE are closely related to the AXEs CE7 originated from Bacillus species, whereas they are distantly located from the fungal AXEs without the CCD activity. The amino acid sequence alignment revealed that three separate amino acid sequence motifs (RGQ, GxSQGG, and HE) are highly conserved in LaAXE, BhAXE, and the other known AXE CE7 from Bacillus pumilus [23] (Fig. 6). These three conserved motifs with the appropriate spacing have been defined as the signature features of CE family 7 enzymes [29]. In addition, the putative catalytic triad residues (Ser178, Asp268, and His298 in LaAXE) were wellconserved in these three AXEs, which were commonly found from the AXEs CE7 with the CCD activity.

Based on the amino acid sequence of LaAXE, the threedimensional model structure was constructed via the Phyre $^{2}$ 3D-modelling server [30]. LaAXE shares the highest structural similarity (41\%) with the Thermoanaerobacterium AXE (PDB ID: 3FCY). The monomeric model of LaAXE

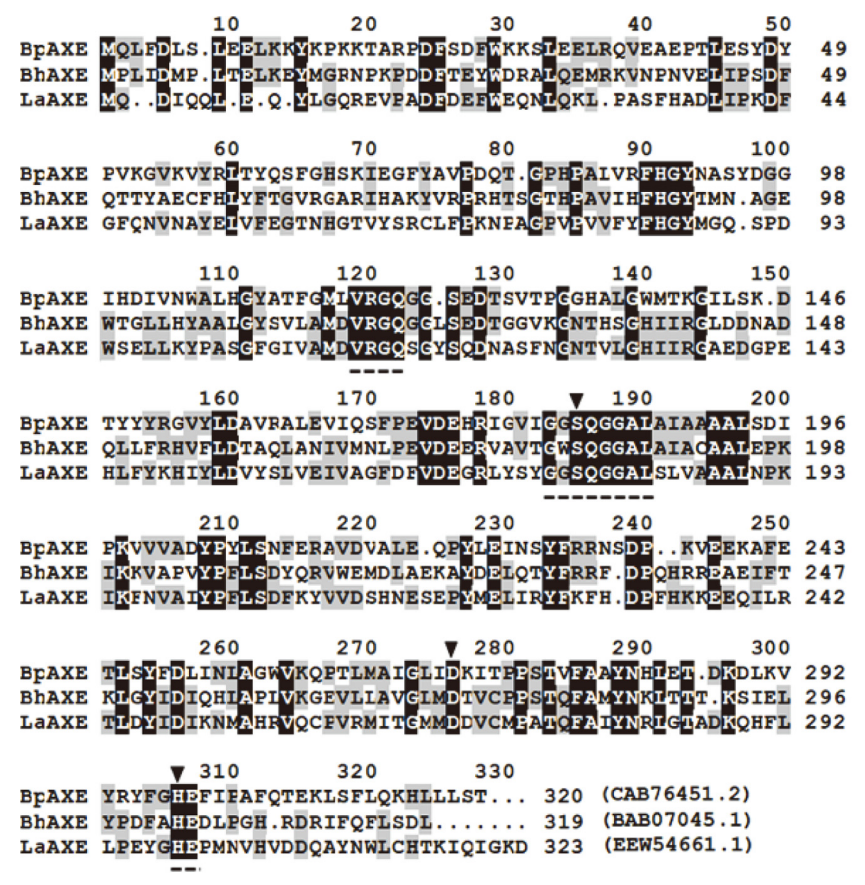

Fig. 6. Amino acid sequence alignment among bacterial AXEs CE family 7 .

The fully conserved amino acid residues were shaded in black and the catalytic triad (Ser-Asp-His) is indicated by arrowheads. The conserved residues related to $C C D$ activity are shown by dashed underlines. BpAXE from Bacillus pumilus; BhAXE from B. halodurans; LaAXE from Lactobacillus antri.

possesses the $\alpha / \beta$-hydrolase fold consisting of the central eight paralleled $\beta$-sheets flanked by $\alpha$-helices, which is highly conserved in the known structures of AXEs CE7 from Thermotoga maritima (5FDF), Thermoanaerobacterium sp. (3FCY), B. pumilus (2XLB), and B. subtilis (1L7A). These AXEs share very similar quaternary structures of homohexameric oligomer consisting of a trimer of dimers [11, 31].

According to the primary and tertiary structure analyses, LaAXE and BhAXE could be classified as the new members of AXE CE family 7. Even though the activity towards 7ACA was not so high, the first AXE CE7 with broad substrate specificity was functionally characterized from Lb. antri, a strain of lactic acid bacteria. It possesses the considerable deacetylating activities against beechwood xylan and its hydrolysates, which can promote the successive actions of $\beta$-xylanase. When the modes of action of LaAXE are understood in detail, it will be one of the better candidates for the enzymatic production of antibiotics and prebiotic carbohydrate materials. 


\section{Acknowledgments}

This work was supported by the National Research Foundation (NRF2013R1A1A2063848 and NRF-2017M3C 1B5019292) of the Ministry of Science, ICT and Future Planning, Republic of Korea.

\section{Conflict of Interest}

The authors have no financial conflicts of interest to declare.

\section{References}

1. Huang YC, Chen GH, Chen YF, Chen WL, Yang CH. 2010. Heterologous expression of thermostable acetylxylan esterase gene from Thermobifida fusca and its synergistic action with xylanase for the production of xylooligosaccharides. Biochem. Biophys. Res. Commun. 400: 718-723.

2. Zheng F, Huang J, Yin Y, Ding S. 2013. A novel neutral xylanase with high SDS resistance from Volvariella volvacea: characterization and its synergistic hydrolysis of wheat bran with acetyl xylan esterase. J. Ind. Microbiol. Biotechnol. 40: 1083-1093.

3. Hettiarachchi SA, Kwon YK, Lee Y, Jo E, Eom TY, Kang YH, et al. 2019. Characterization of an acetyl xylan esterase from the marine bacterium Ochrovirga pacifica and its synergism with xylanase on beechwood xylan. Microb. Cell Fact. 18: 122.

4. Malgas S, Mafa MS, Mkabayi L, Pletschke B. 2019. A mini review of xylanolytic enzymes with regards to their synergistic interactions during hetero-xylan degradation. World J. Microbiol. Biotechnol. 35(12): 187.

5. Biely P. 2012. Microbial carbohydrate esterases deacetylating plant polysaccharides. Biotechnol. Adv. 30: 1575-1588.

6. Poeker SA, Geirnaert A, Berchtold L, Greppi A, Krych L, Steinert RE, et al. 2018. Understanding the prebiotic potential of different dietary fibers using an in vitro continuous adult fermentation model (PolyFermS). Sci. Rep. 8: 4318.

7. Adesioye FA, Makhalanyane TP, Biely P, Cowan DA. 2016. Phylogeny, classification and metagenomic bioprospecting of microbial acetyl xylan esterases. Enzyme Microb. Technol. 93-94: 79-91.

8. Sista Kameshwar AK, Qin W. 2018. Understanding the structural and functional properties of carbohydrate esterases with a special focus on hemicellulose deacetylating acetyl xylan esterases. Mycology 9: 273-295.

9. Mitsushima K, Takimoto A, Sonoyama T, Yagi S. 1995. Gene cloning, nucleotide sequence, and expression of a cephalosporinC deacetylase from Bacillus subtilis. Appl. Environ. Microbiol. 61: 2224-2229.

10. Degrassi G, Kojic M, Ljubijankic G, Venturi V. 2000. The acetyl xylan esterase of Bacillus pumilus belongs to a family of esterases with broad substrate specificity. Microbiology 146: 1585-1591.

11. Levisson M, Han GW, Deller MC, Xu Q, Biely P, Hendriks $S$, et al. 2012. Functional and structural characterization of a thermostable acetyl esterase from Thermotoga maritima. Proteins 80: 1545-1559.

12. Tian Q, Song P, Jiang L, Li S, Huang H. 2014. A novel cephalosporin deacetylating acetyl xylan esterase from Bacillus subtilis with high activity toward cephalosporin C and 7-aminocephalosporanic acid. Appl. Microbiol. Biotechnol. 98: 2081-2089.

13. Sonawane VC. 2006. Enzymatic modifications of cephalosporins by cephalosporin acylase and other enzymes. Crit. Rev. Biotechnol. 26: 95-120.

14. Liu Y, Gong G, Zhu C, Zhu B, Hu Y. 2010. Environmentally safe production of 7-ACA by recombinant Acremonium chrysogenum. Curr. Microbiol. 61: 609-614.

15. Viborg AH, Sørensen KI, Gilad O, Steen-Jensen DB, Dilokpimol A, Jacobsen S, et al. 2013. Biochemical and kinetic characterisation of a novel xylooligosaccharide-upregulated GH43 $\beta$-D-xylosidase/ $\alpha$-L-arabinofuranosidase (BXA43) from the probiotic Bifidobacterium animalis subsp. lactis BB-12. AMB Express 3: 56.

16. Maria A, Margarita T, IIlia I, Iskra I. 2014. Gene expression of enzymes involved in utilization of xylooligosaccharides by Lactobacillus strains. Biotechnol. Biotechnol. Equip. 28: 941-948.

17. Park JM, Han NS, Kim TJ. 2007. Rapid detection and isolation of known and putative $\alpha$-L-arabinofuranosidase genes using degenerate PCR primers. J. Microbiol. Biotechnol. 17: 481-489.

18. Khandeparker R, Jalal T. 2015. Xylanolytic enzyme systems in Arthrobacter sp. MTCC 5214 and Lactobacillus sp. Biotechnol. Appl. Biochem. 62: 245-254.

19. Roos S, Engstrand L, Jonsson H. 2005. Lactobacillus gastricus sp. nov., Lactobacillus antri sp. nov., Lactobacillus kalixensis sp. nov. and Lactobacillus ultunensis sp. nov., isolated from human stomach mucosa. Int. J. Syst. Evol. Microbiol. 55: 77-82.

20. Takami H, Nakasone K, Takaki Y, Maeno G, Sasaki R, Masui $\mathrm{N}$, et al. 2000. Complete genome sequence of the alkaliphilic bacterium Bacillus halodurans and genomic sequence comparison with Bacillus subtilis. Nucleic Acids Res. 28: 4317-4331.

21. Margolles-Clark E, Tenkanen M, Söderlund H, Penttilä M. 1996. Acetyl xylan esterase from Trichoderma reesei contains an active-site serine residue and a cellulose-binding domain. Eur. J. Biochem. 237: 553-560.

22. Koseki T, Miwa Y, Akao T, Akita O, Hashizume K. 2006. An Aspergillus oryzae acetyl xylan esterase: molecular cloning and characteristics of recombinant enzyme expressed in Pichia pastoris. J. Biotechnol. 121: 381-389.

23. Krastanova I, Guarnaccia C, Zahariev S, Degrassi G, Lamba D. 2005. Heterologous expression, purification, crystallization, 
X-ray analysis and phasing of the acetyl xylan esterase from Bacillus pumilus. Biochim. Biophys. Acta 1748: 222-230.

24. Drzewiecki K, Angelov A, Ballschmiter M, Tiefenbach KJ, Sterner R, Liebl W. 2010. Hyperthermostable acetyl xylan esterase. Microb. Biotechnol. 3: 84-92.

25. Shao W, Wiegel J. 1995. Purification and characterization of two thermostable acetyl xylan esterases from Thermoanaerobacterium sp. strain JW/SL-YS485. Appl. Environ. Microbiol. 61: 729-733.

26. Yang CH, Liu WH. 2008. Purification and properties of an acetylxylan esterase from Thermobifida fusca. Enzyme Microb. Technol. 42: 181-186.

27. Park SH, Yoo W, Lee CW, Jeong CS, Shin SC, Kim HW, et al. 2018. Crystal structure and functional characterization of a cold-active acetyl xylan esterase (PbAcE) from psychrophilic soil microbe Paenibacillus sp. PLoS One 13: e0206260.
28. Velikodvorskaya TV, Volkov IY, Vasilevko VT, Zverlov VV, Piruzian ES. 1997. Purification and some properties of Thermotoga neapolitana thermostable xylanase B expressed in E. coli cells. Biochemistry (Mosc) 62: 66-70.

29. Vincent F, Charnock SJ, Verschueren KH, Turkenburg JP, Scott DJ, Offen WA, et al. 2003. Multifunctional xylooligosaccharide/cephalosporin C deacetylase revealed by the hexameric structure of the Bacillus subtilis enzyme at 1.9 ̊ resolution. J. Mol. Biol. 330: 593-606.

30. Kelley LA, Mezulis S, Yates CM, Wass MN, Sternberg MJ. 2015. The Phyre2 web portal for protein modeling, prediction and analysis. Nat. Protoc. 10: 845-858.

31. Singh MK, Manoj N. 2016. An extended loop in CE7 carbohydrate esterase family is dispensable for oligomerization but required for activity and thermostability. J. Struct. Biol. 194: 434-445. 